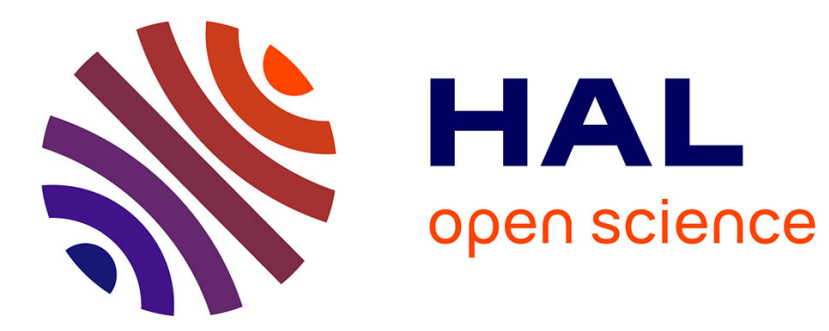

\title{
Elastocaloric effect dependence on pre-elongation in natural rubber
}

Zhongjian Xie, Gaël Sebald, Daniel Guyomar

\section{To cite this version:}

Zhongjian Xie, Gaël Sebald, Daniel Guyomar. Elastocaloric effect dependence on pre-elongation in natural rubber. Applied Physics Letters, 2015, 107 (8), 10.1063/1.4929395 . hal-01769266

\section{HAL Id: hal-01769266 \\ https://hal.science/hal-01769266}

Submitted on 6 May 2020

HAL is a multi-disciplinary open access archive for the deposit and dissemination of scientific research documents, whether they are published or not. The documents may come from teaching and research institutions in France or abroad, or from public or private research centers.
L'archive ouverte pluridisciplinaire HAL, est destinée au dépôt et à la diffusion de documents scientifiques de niveau recherche, publiés ou non, émanant des établissements d'enseignement et de recherche français ou étrangers, des laboratoires publics ou privés. 


\title{
Elastocaloric effect dependence on pre-elongation in natural rubber
}

\author{
Zhongjian Xie, Gael Sebald, ${ }^{\text {a) }}$ and Daniel Guyomar \\ University de Lyon, INSA-Lyon, LGEF EA682, 8 rue de la Physique, F-69621 Villeurbanne, France
}

\begin{abstract}
In the context of solid-state-cooling, the elastocaloric effect offers a very large controlled entropy change based in low-cost polymers, especially natural rubber which is environmentally friendly. However, large elastocaloric activity requires large elongation $(>5)$, which makes this material impractical for cooling systems due to the large change in sample's area. By performing a pre-elongation, area change is limited, and $\beta=-\partial \gamma / \partial \lambda$ (where $\gamma$ is the specific entropy and $\lambda$ is the elongation) is larger. The highest $\beta$ value is obtained when pre-elongation is right before (at the "eve") the onset of the strain-induced crystallization, which is also interpreted in the view of molecular conformation. Experimental results obtained on a natural rubber sample showed an adiabatic temperature change of $4.3^{\circ} \mathrm{C}$ for pre-elongation of 4 with further elongation of 4 (true strain change of $69 \%$ ). Furthermore, the entropy exhibits a quasi-linear dependence on elongation, and the $\beta$ value is found to be $6400 \mathrm{~J} \mathrm{~K}^{-1} \mathrm{~m}^{-3}$.
\end{abstract}

The elastocaloric effect, with other caloric effects such as magnetocaloric, barocaloric, and electrocaloric, can be used for solid cooling systems, and is thus expected to renew present cooling devices that are based on the vapor compression of hazardous gases. Electrocaloric materials are limited by their dielectric strength, ${ }^{1,2}$ whereas magnetocaloric material are mostly made of rare earth elements, ${ }^{3}$ which are hardly sustainable. Shape memory alloys are conventional elastocaloric and barocaloric materials. ${ }^{4-6}$ For example, $\mathrm{Ni}-\mathrm{Ti}$ alloy exhibits a very large elastocaloric effect. ${ }^{7}$ The elastocaloric effect of rubber (called the Gough-Joule effect) was first observed by Gough and further investigated by Joule. ${ }^{8}$ Natural rubber (NR) can be chosen as a solid cooling material due to its non-toxicity, sustainability, and low cost. However, to obtain large temperature variations, the NR needs to be stretched to several times its initial length. Like in active magnetic regenerator (AMR), where a porous bed of magnetic refrigerant material is used to transfer heat efficiently, ${ }^{9}$ theoretical calculation of elastocaloric cooling system was developed by Tušek et al. ${ }^{10}$ in which an active regenerative cycle was demonstrated. A similar elastocaloric regenerator by using NR material can be conducted. A NR tube is chosen as elastocaloric regenerator. It has double functions, one is for the elastocaloric effect and the other is for the passage of heat transfer fluid. The NR tube should have enough area at the extension and retraction state. When the retraction state is assured to have an enough area, the extension state would be too large. These factors make NR impractical in the design of compact cooling devices. In this background, a pre-elongation effect is investigated. This paper describes the elastocaloric coefficient $\beta=-\partial \gamma / \partial \lambda$ dependence on elongation, where $\gamma$ represents the specific entropy and $\lambda$ represents the elongation. The elastocaloric effect of NR with and without pre-elongation was measured and compared, and the underlying mechanism related to the strain-induced crystallization (SIC) is discussed. In this

a)gael.sebald@insa-lyon.fr paper, two deformation variables are used, one is elongation and the other is true strain. The elongation variable $\lambda=l / l_{0}$ is defined as the ratio of the elongated length $l$ of the sample to the initial length $l_{0}$. The true strain variable is the logarithm of the elongation. The true strain variable is used for highlighting the benefit of pre-strain condition from view of material. The elongation variable might however be more easily understood for large strain levels. The experimental part of the study consisted of tensile tests using an Ironless Linear Motor (XM-550, Newport, New York) and temperature measurements. ${ }^{11}$ The cross section area of NR film (Xinyinte Rubber Products Co., Ltd.) is $15 \mathrm{~mm} \times 70 \mu \mathrm{m}$ and the original length is $10 \mathrm{~mm}$. The temperature was detected by an Infrared Camera (NEC G-120, NEC, Japan) through a hole. Infrared (IR) images were recorded at a frame rate of $10 \mathrm{~Hz}$, and the noise level of the temperature information from the IR camera was $\pm 0.1{ }^{\circ} \mathrm{C} .{ }^{12}$

The stress-elongation behavior with elongation rate of $0.028 \mathrm{~s}^{-1}$ is shown in Fig. 1. The stress-elongation hysteresis is relatively stable after 20 cycles. Part of the mechanical

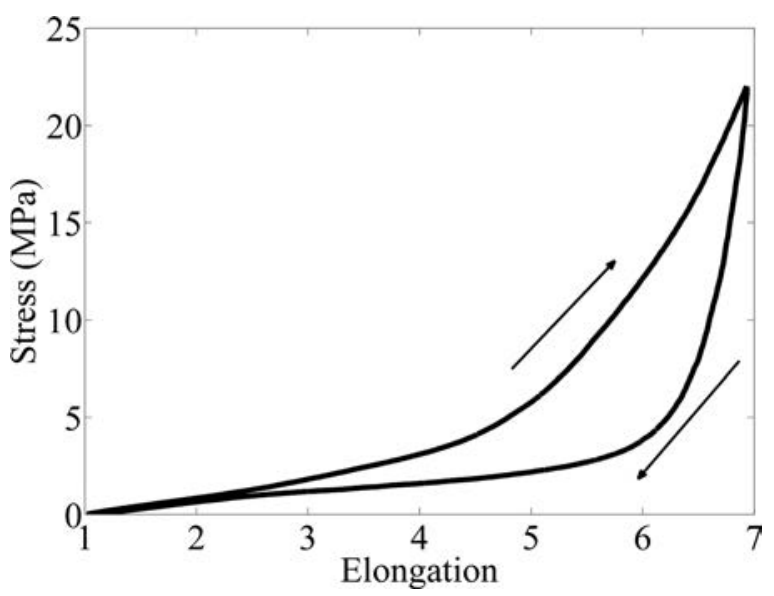

FIG. 1. Stress-elongation curve of relative stable state after 20 cycles at elongation rate of $0.028 \mathrm{~s}^{-1}$. 
hysteresis may be due to SIC. ${ }^{13-15}$ The stress upturn during loading process is usually considered to be due to the dominant reinforcement effect of SIC. More deeply, it can alternatively be interpreted as the distortion of backbone bond angles as a network chain is extended beyond its contour length. ${ }^{16}$ The lower stress during unloading process is due to the larger degree of SIC, which shows the dominant stress relaxation effect of SIC. Another part of mechanical hysteresis may however be due to the chain rupture during stretching process. ${ }^{16}$

For our sample, the adiabatic limit elongation rate was measured to be around $10 \mathrm{~s}^{-1}$. In the experiment, the elongation rate of $30 \mathrm{~s}^{-1}$ was chosen for measuring the temperature change. The larger adiabatic limit elongation rate compared with Ni-Ti alloy ${ }^{7,17}$ is because of the smaller thickness of NR. Fig. 2 displays a typical time profile of the temperature with time. The difference of measured temperature change between loading and unloading can be explained by the different kinetics properties of SIC and melting. ${ }^{13,18,19}$ SIC requiring time to be completed, part of the SIC occurs while the elongation is constant. ${ }^{20}$ This part of SIC will not be measured due to heat loss with outer medium. In the unloading process, the melting is much faster than SIC. So, nearly all the SIC will melt during the unloading process and contribute to temperature decrease. As a result, there is a difference of temperature change in the measurement; even the total entropy change should be the same during loading and unloading. This result was also observed by Sakata at larger elongation. $^{21}$

Network chain density of the sample was estimated according to Eq. (1) based on the standard theory of rubber elasticity $^{22}$

$$
\sigma=N k T\left(\lambda-\lambda^{-2}\right)
$$

where $\sigma$ is the stress, $N$ is network chain density, $k$ is the Boltzmann constant, $T$ is the absolute temperature, and $\lambda$ is the elongation. The material has $N$ value of $2.8 \times 10^{-4} \mathrm{~mol} \mathrm{~cm}^{-3}$ that is calculated from the initial region of stress-elongation curve, which is of the same order of magnitude as published material. $^{23}$

The adiabatic temperature variation $\Delta T$ is proportional to the isothermal elastocaloric entropy variation $\Delta \gamma$

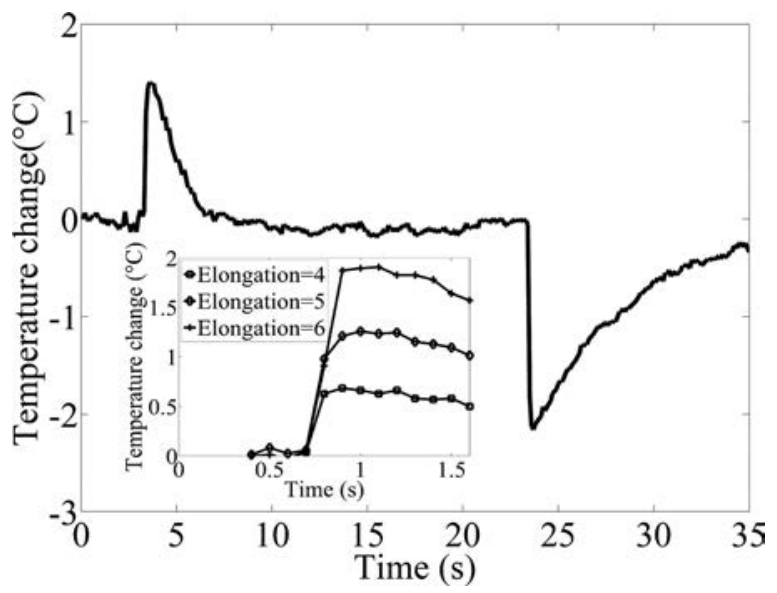

FIG. 2. Typical temperature-time signal of rubber with an elongation of 5 recorded as determined by an infrared imaging camera. Inset shows enlargement of temperature increase at the time of elongation step.

$$
T_{0} \times\left.\Delta \gamma\right|_{T=T_{0}}=-c \times\left.\Delta T\right|_{\gamma=\gamma_{0}},
$$

where $T_{0}=295 \mathrm{~K}$ is the temperature at which the elastocaloric effect is observed, $\gamma_{0}$ is the entropy when undergoing an adiabatic measurement, and $c=1.8 \times 10^{6} \mathrm{~J} \mathrm{~K}^{-1} \mathrm{~m}^{-3}$ is an estimation of specific heat of $\mathrm{NR},{ }^{24}$ and assumed to be independent of the elongation and temperature. So,

$$
\Delta \gamma=-\frac{c}{T_{0}} \Delta T
$$

The elastocaloric coefficient $\beta=-\partial \gamma / \partial \lambda$ was used to evaluate the elastocaloric conversion effectiveness of elastocaloric materials. It can be calculated by deriving $\Delta T$ versus $\lambda$ function

$$
\beta=\frac{c}{T_{0}} \cdot \frac{\partial(\Delta T)}{\partial \lambda}
$$

According to the new paradigm for the molecular basis of rubber elasticity, ${ }^{25}$ three extension regimes should be identified: low, medium, and high. In the low extension, the rubber elasticity is associated with the distribution of end-toend distances of molecular kinks. ${ }^{26}$ In the medium extension, the rubber elasticity is caused by the rotational conformations of (poly)isoprene molecules. ${ }^{27}$ In the high extension, the rubber elasticity is caused by the purely enthalpic chain stretching. ${ }^{16}$

As Hanson and Martin have demonstrated, bond rupture occurs at relatively large strains for polybutadiene, which implies that purely enthalpic chain stretching must commence well before tensile failure occurs. ${ }^{16}$ This phenomenon can also be interpreted in the view of SIC theory. SIC in the draw direction relaxes the remaining amorphous chains. ${ }^{28-30}$ So, SIC decreases the stress and increases the strain at break. $^{31}$ The breakage of chain is due to the saturation of SIC as the molecules may have no room to move to the minimum energy position at high strains. ${ }^{31}$ At the same time, it has been extensively evidenced that SIC of NR occurs at around elongation of 4 at $25^{\circ} \mathrm{C} .{ }^{13,23,32,33}$ So, it can be proved that the upturn of temperature change from elongation of 4 is due to the enthalpic process by both the theories of Hanson and Martin at high extension ${ }^{16}$ and the SIC. Also, in Fig. 2, the difference of measured temperature change between extension and retraction can be explained by the part of SIC of post-stretch ${ }^{20}$ (more detail in the description part of Fig. 2). The temperature change of post-stretch process cannot be explained by the entropy elasticity because the instantaneous coil-stretch transition proposed by De Gennes. ${ }^{34}$ Mitchell and Meier ${ }^{35}$ also show that SIC is dominant for temperature change. Upon the stretching of NR, irreversible entropy variation may occur due to the rupturing of network chains. ${ }^{36}$ In our work, only the temperature decrease upon unloading was presented in order to avoid any irreversible effect. To get a high elastocaloric coefficient, a preelongation can be applied. The low elastocaloric coefficient region can be skipped and go directly into the high elastocaloric coefficient region. In the experiments, a pre-elongation of $3,4,5$, and 6 are applied to the NR sample and the adiabatic temperature change is measured upon further elongation (Fig. 3). The true strain variable calculated by the logarithm 


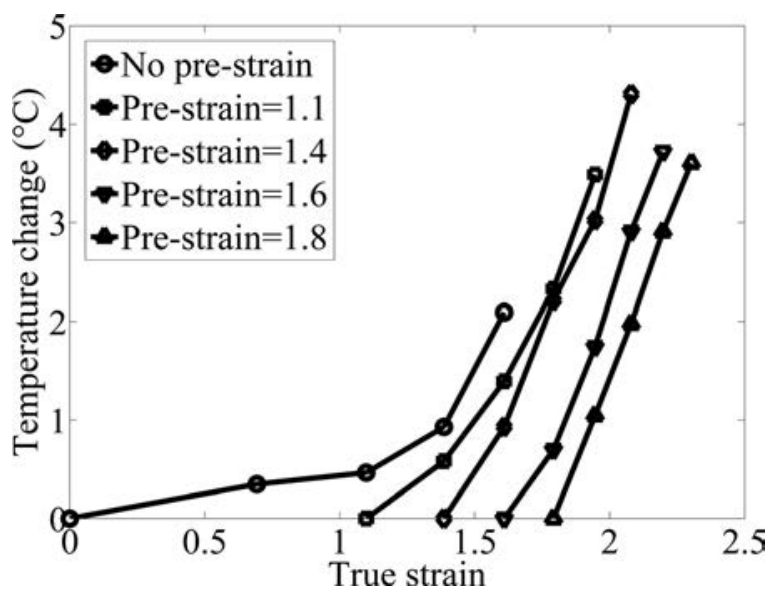

FIG. 3. Comparison of temperature change of no pre-strain deformation and pre-strain deformation.

of the elongation is used for highlighting the benefit of prestrain condition. The pre-elongations $(3,4,5$, and 6$)$ corresponds to pre-strains of $1.1,1.4,1.6$, and 1.8 respectively. It is obviously shown through true strain variable that the slope of the temperature change is enhanced by the pre-strain condition. For pre-elongation of 4 , further elongation to 8 (corresponding to an additional true strain of 0.69) leads to a temperature variation of $4.3^{\circ} \mathrm{C}$. Considering the pre-elongated sample, its relative elongation $l / l_{\text {pre-elongated }}$ would then be only 2.

From the previous experiments, the elastocaloric coefficient is calculated for the different pre-elongations. As the temperature change depends almost linearly on the elongation, we provide with a single value of coefficient for each pre-elongation as shown in Fig. 4. For pre-elongation experiments, it is observed that the $\beta$ value is larger than no pre-elongation stretching. Comparing the different preelongations, it is worth to notice that the $\beta$ value obtains a maximum value when a pre-elongation of 4 is applied at the eve of SIC. This is the same mechanism as for the stress upturn: the distortion of backbone bond angles as a network chain is extended beyond its contour length. ${ }^{16}$ When preelongation is located at SIC region, the $\beta$ value decreases again.

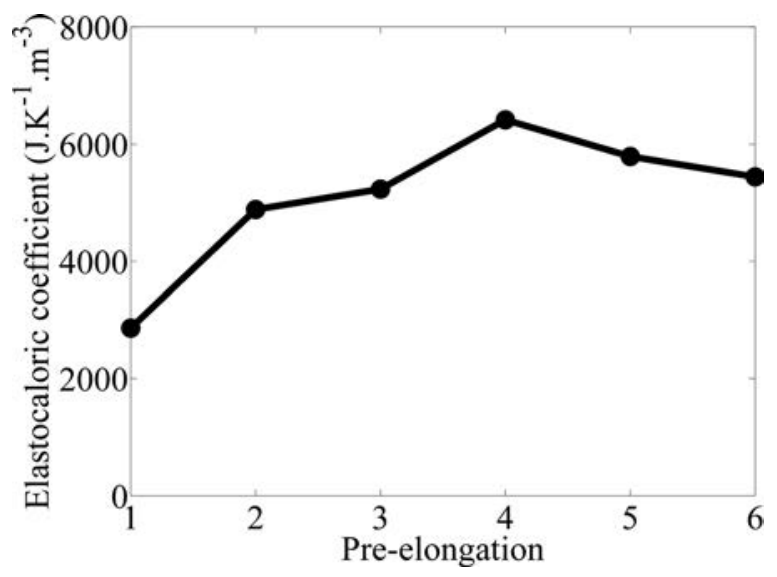

FIG. 4. Different elastocaloric coefficients at different pre-elongations and no pre-elongation conditions. The pre-elongation of 1 indicates the no preelongation condition.
In case of no pre-elongation stretching, the maximum value of $\beta$ can be estimated in the region of $4<\lambda<8$ with $\beta \sim 8100 \mathrm{~J} \mathrm{~K}^{-1} \mathrm{~m}^{-3}$, which is larger than pre-elongation condition (maximum around 6400). In order to better interpret SIC mechanisms at different elongations, the NR sample is stretched to different elongations and is kept. In the inset of Fig. 2 is shown the temperature signal for an enlarged time scale. It is observed a much larger creep effect for an elongation of 5. This is because of the longer SIC incubation time ${ }^{37,38}$ and crystallite nuclei have more room to grow at onset of SIC comparing with the high elongation. Accompanying the temperature creep, stress relaxation occurs. ${ }^{20}$ SIC, taking up less space in the lateral direction ${ }^{23,39}$ and relaxing the remaining amorphous chain in the stretching direction, ${ }^{28-30}$ approaches the one of minimum potential energy. ${ }^{40}$ This phenomenon corresponds to the theoretical result of Hanson et al.: ${ }^{27}$ the poly-isoprene configurations with shorter end-to-end distances tend to have higher energies. Isoprene units along the chain backbone are mechanically forced from their equilibrium distributions to a smaller subset of states, restricted to more linear conformations with the greatest end-to-end distances. ${ }^{27}$

As a consequence, further elongation from a pre-elongation of 5 results in lower degree SIC than a no pre-elongation process, the material being closer from the saturation of SIC, and is the reason for the decrease of the $\beta$ value when pre-elongations locate at SIC region.

Corresponding to the thermal response, the stress response of different pre-elongations condition is also measured. After the holding time at constant elongation, it is increased abruptly up to elongation of 7 for all tested pre-elongations. When the final elongation is reached, the final stress is recorded. In Fig. 5, the stress between pre-elongation and final elongation is compared for the different pre-elongation conditions. The maximum stress is obtained between elongation of 5 and 7. As crystallite is a big network point binding a large number of chains $^{41}$ and thus divides the original chain into short one, this reduces the limit of extensibility and contributes to the stress. As discussed before, the molecular chain can crystallize to a large extent at onset of SIC, which means one crystallite at onset of SIC can bind more chains than one crystallite at high

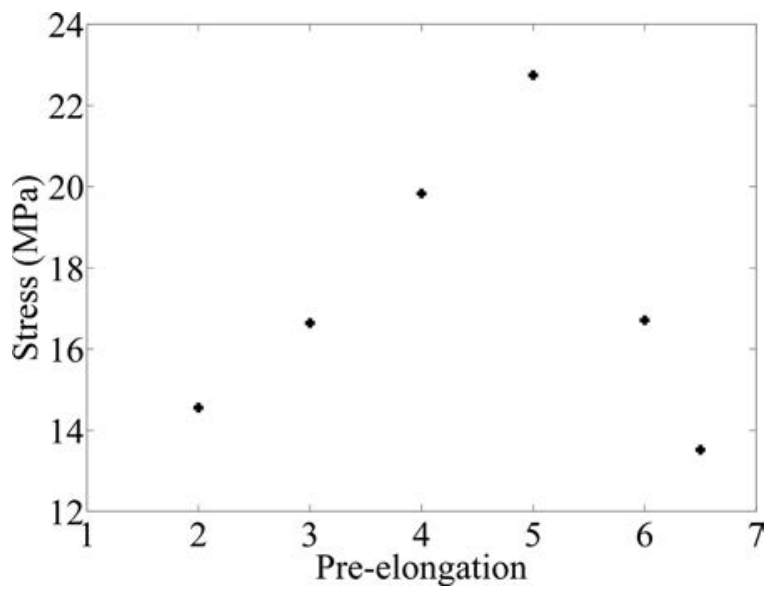

FIG. 5. The stress between different pre-elongations (from elongation of 2-6.5) and the same final elongation of 7. The maximum stress occurs between elongation of 5 and 7 . The stress does not decrease from pre-elongation of 2-5 even the decreasing strain change. 
elongation. Thus, the SIC can contribute more to the stress in the further elongation when the pre-elongation is at the onset of SIC. The large increase of the stress is then associated with a larger crystallization at the pre-elongated state, but limiting further crystallization upon elongation up to 7 . So, even there is a maximum entropic stress between elongation of 5 and 7 , the temperature change is lower compared to the elongation from 4 to 7 . The opposite tendency of stress and temperature shows that the entropy change upon elongation is mainly due to the crystallization and associated enthalpy of melting, and the entropic elasticity contribution is secondary. Considering the large mechanical energy input but small temperature change output at pre-elongation of 5, pre-elongation of 5 is not recommended for application.

As a conclusion, the effect of pre-elongation on the elastocaloric effect is investigated. Compared with the no preelongation deformation, the low elastocaloric coefficient region can be skipped and go directly into the high elastocaloric coefficient region due to SIC by applying preelongation. Especially, the elastocaloric coefficient obtains the highest value when pre-elongation is applied at eve of SIC, whereas elastocaloric coefficient decreases again when pre-elongation goes into SIC region. Also, the stress input is the maximum when pre-elongation is applied at onset of SIC. The eve of SIC should then be chosen as the preelongation rather than onset of SIC. For application, any irreversible process (overall damaging) should be avoided due to large elongation, ${ }^{36}$ since it results in overall heating of the sample and is too close to the toughness limit. Further investigation on fatigue property of different pre-elongations and final elongations is necessary for potential application. Furthermore, according to Tušek et al., ${ }^{10}$ a similar elastocaloric regenerator by using NR material can be conducted.

${ }^{1}$ M. Valant, Prog. Mater. Sci. 57, 980 (2012).

${ }^{2}$ X. Yin, J.-F. Capsal, and D. Guyomar, Appl. Phys. Lett. 104, 052913 (2014).

${ }^{3}$ B. Yu, M. Liu, P. W. Egolf, and A. Kitanovski, Int. J. Refrig. 33, 1029 (2010).

${ }^{4}$ L. Mañosa, A. Planes, and M. Acet, J. Mater. Chem. A 1, 4925 (2013).

${ }^{5}$ E. Bonnot, R. Romero, L. Mañosa, E. Vives, and A. Planes, Phys. Rev. Lett. 100, 125901 (2008).

${ }^{6}$ L. Mañosa, D. González-Alonso, A. Planes, E. Bonnot, M. Barrio, J.-L. Tamarit, S. Aksoy, and M. Acet, Nat. Mater. 9, 478 (2010).

${ }^{7}$ J. Tušek, K. Engelbrecht, L. P. Mikkelsen, and N. Pryds, J. Appl. Phys. 117, 124901 (2015)
${ }^{8}$ G. A. Holzapfel and J. C. Simo, Comput. Methods Appl. Mech. Eng. 132, 17 (1996).

${ }^{9}$ V. K. Pecharsky and K. A. Gschneidner, Jr., J. Magn. Magn. Mater. 200, 44 (1999).

${ }^{10}$ J. Tušek, K. Engelbrecht, R. Millán-Solsona, L. Mañosa, E. Vives, L. P. Mikkelsen, and N. Pryds, Adv. Energy Mater. 5, 1500361 (2015).

${ }^{11}$ D. Guyomar, Y. Li, G. Sebald, P. J. Cottinet, B. Ducharne, and J. F. Capsal, Appl. Therm. Eng. 57, 33 (2013).

${ }^{12}$ G. Sebald, L. Seveyrat, J. F. Capsal, P. J. Cottinet, and D. Guyomar, Appl. Phys. Lett. 101, 022907 (2012).

${ }^{13}$ S. Trabelsi, P. A. Albouy, and J. Rault, Macromolecules 36, 7624 (2003).

${ }^{14}$ I. S. Choi and C. M. Roland, Rubber Chem. Technol. 70, 202 (1997).

${ }^{15}$ J. E. Mark, E. Burak, and E. Mike Roland, The Science and Technology of Rubber (Academic press, Oxford, 2013).

${ }^{16}$ D. E. Hanson and R. L. Martin, J. Chem. Phys. 130, 064903 (2009).

${ }^{17}$ H. Ossmer, F. Lambrecht, M. Gültig, C. Chluba, E. Quandt, and M. Kohl, Acta Mater. 81, 9 (2014).

${ }^{18}$ J. R. Samaca Martinez, J. B. Le Cam, X. Balandraud, E. Toussaint, and J. Caillard, Polymer 54, 2717 (2013).

${ }^{19}$ K. Brüning, K. Schneider, S. V. Roth, and G. Heinrich, Macromolecules 45, 7914 (2012).

${ }^{20}$ M. Tosaka, D. Kawakami, K. Senoo, S. Kohjiya, Y. Ikeda, S. Toki, and B. S. Hsiao, Macromolecules 39, 5100 (2006).

${ }^{21}$ A. Sakata, N. Suzuki, Y. Higashiura, T. Matsuo, and T. Sato, J. Therm. Anal. Calorim. 113, 1555 (2013).

${ }^{22}$ L. R. G. Treloar, The Physics of Rubber Elasticity (Oxford university press, Oxford, 1975).

${ }^{23}$ M. Tosaka, S. Murakami, S. Poompradub, S. Kohjiya, Y. Ikeda, S. Toki, I. Sics, and B. S. Hsiao, Macromolecules 37, 3299 (2004).

${ }^{24}$ P. Ortiz-Serna, R. Díaz-Calleja, and M. J. Sanchis, J. Appl. Polym. Sci. 128, 2269 (2013).

${ }^{25}$ D. E. Hanson and J. L. Barber, Contemp. Phys. 56, 319 (2015).

${ }^{26}$ D. E. Hanson, J. Chem. Phys. 134, 064906 (2011).

${ }^{27}$ D. E. Hanson, J. L. Barber, and G. Subramanian, J. Chem. Phys. 139, 224906 (2013).

${ }^{28}$ P. A. Albouy, A. Vieyres, R. Pérez-Aparicio, O. Sanséau, and P. Sotta, Polymer 55, 4022 (2014).

${ }^{29}$ J. Rault, J. Marchal, P. Judeinstein, and P. A. Albouy, Eur. Phys. J. E 21, 243 (2006).

${ }^{30}$ P. J. Flory, J. Chem. Phys. 15, 397 (1947).

${ }^{31}$ S. Toki, B. S. Hsiao, S. Amnuaypornsri, and J. Sakdapipanich, Polymer 50, 2142 (2009).

${ }^{32}$ N. Candau, R. Laghmach, L. Chazeau, J.-M. Chenal, C. Gauthier, T. Biben, and E. Munch, Macromolecules 47, 5815 (2014).

${ }^{33}$ S. Toki, T. Fujimaki, and M. Okuyama, Polymer 41, 5423 (2000).

${ }^{34}$ P. G. De Gennes, J. Chem. Phys. 60, 5030 (1974).

${ }^{35}$ J. C. Mitchell and D. J. Meier, Rubber Chem. Technol. 42, 1420 (1969).

${ }^{36}$ D. E. Hanson, J. Chem. Phys. 131, 224904 (2009).

${ }^{37}$ N. Candau, L. Chazeau, J. M. Chenal, C. Gauthier, J. Ferreira, E. Munch, and C. Rochas, Polymer 53, 2540 (2012).

${ }^{38}$ B. Huneau, Rubber Chem. Technol. 84, 425 (2011).

${ }^{39}$ C. W. Bunn, Rubber Chem. Technol. 15, 742 (1942).

${ }^{40}$ G. Natta and P. Corradini, Nuovo Cimento 15, 40 (1960).

${ }^{41}$ S. Toki, J. Che, L. Rong, B. S. Hsiao, S. Amnuaypornsri, A. Nimpaiboon, and J. Sakdapipanich, Macromolecules 46, 5238 (2013). 